\title{
eROSITA on SRG
}

\author{
Peter Predehl*, Hans Böhringer*, Hermann Brunner*, Marcella Brusa*, \\ Vadim Burwitz*, Nico Cappelluti* Evgeniy Churazov ${ }^{\dagger}$, Konrad Dennerl*, \\ Michael Freyberg*, Peter Friedrich*, Alexis Finoguenov*, Günther \\ Hasinger**, Eckhard Kendziorra ${ }^{\ddagger}$, Ingo Kreykenbohm, Christian Schmid, \\ Jörn Wilms ${ }^{\S}$, Georg Lamer ${ }^{\mathbb{I}}$, Norbert Meidinger*, Martin Mühlegger*, \\ Mikhail Pavlinsky ${ }^{\|}$, Jan Robrade ${ }^{\dagger \dagger}$, Andrea Santangelo ${ }^{\ddagger}$, Jürgen Schmitt ${ }^{\dagger \dagger}$, \\ Axel Schwope ${ }^{\mathbb{I}}$, Matthias Steinmetz ${ }^{\mathbb{I}}$, Lothar Strüder*, Rashid Sunyaev ${ }^{\dagger}$ \\ and Chris Tenzer \\ *Max-Planck-Institut für extraterrestrische Physik, D-85741 Garching, Germany \\ ${ }^{\dagger}$ Max-Planck-Institut für Astrophysik, D-85741 Garching, Germany \\ ** Max-Planck-Institut für Plasma Physik, D-85741 Garching, Germany \\ ${ }^{\ddagger}$ Institut für Astronomie und Astrophysik, Abteilung Astronomie, Universität Tübingen, Sand 1, \\ 72076 Tübingen, Germany \\ ${ }^{\S}$ Universit"at Erlangen/Nürnberg, Dr-Remeis-Sternwarte Bamberg, Sternwartstrasse 7, D- \\ Bamberg \\ "Astrophysikalisches Institut Potsdam, An der Sternwarte 16, D-14482 Potsdam, Germany \\ "Space Research Institute Moscow, Russian Federation \\ ${ }^{\dagger}$ Universität Hamburg, Hamburger Sternwarte, Gojenbergsweg 112, 21029 Hamburg, Germany
}

\begin{abstract}
ROSITA (extended ROentgen Survey with an Imaging Telescope Array) is the core instrument on the Russian Spektrum-Roentgen-Gamma (SRG) mission which is scheduled for launch in late 2012. eROSITA is fully approved and funded by the German Space Agency DLR and the Max-Planck-Society.

The design driving science is the detection of 50 - 100 thousands Clusters of Galaxies up to redshift $z \sim 1.3$ in order to study the large scale structure in the Universe and test cosmological models, especially Dark Energy. This will be accomplished by an all-sky survey lasting for four years plus a phase of pointed observations. eROSITA consists of seven Wolter-I telescope modules, each equipped with 54 Wolter-I shells having an outer diameter of $360 \mathrm{~mm}$. This would provide and effective area at $1.5 \mathrm{keV}$ of $\sim 1500 \mathrm{~cm}^{2}$ and an on axis PSF HEW of $15^{\prime \prime}$ which would provide an effective angular resolution of $25^{\prime \prime}-30^{\prime \prime}$. In the focus of each mirror module, a fast frame-store pn-CCD will provide a field of view of $1^{\circ}$ in diameter for an active FOV of $\sim 0.83 \mathrm{deg}^{2}$. At the time of writing the instrument development is currently in phase C/D.
\end{abstract}

Keywords: X-ray astronomy, All-sky Survey, Dark Energy

PACS: $95.55 . \mathrm{Ko}, 97.80 . J p, 98.70 . \mathrm{Qy}$

\section{MISSION OVERVIEW}

The Russian Spectrum-Roentgen-Gamma (SRG) satellite will fly on a medium class platform ("Navigator", Lavochkin Association, Russia). The launch will be in 2012

\footnotetext{
${ }^{1}$ eROSITA is funded equally by the Deutsches Zentrum für Luft und Raumfahrt (DLR) and the MaxPlanck-Gesellschaft zur Förderung der Wissenschaften (MPG).
} 

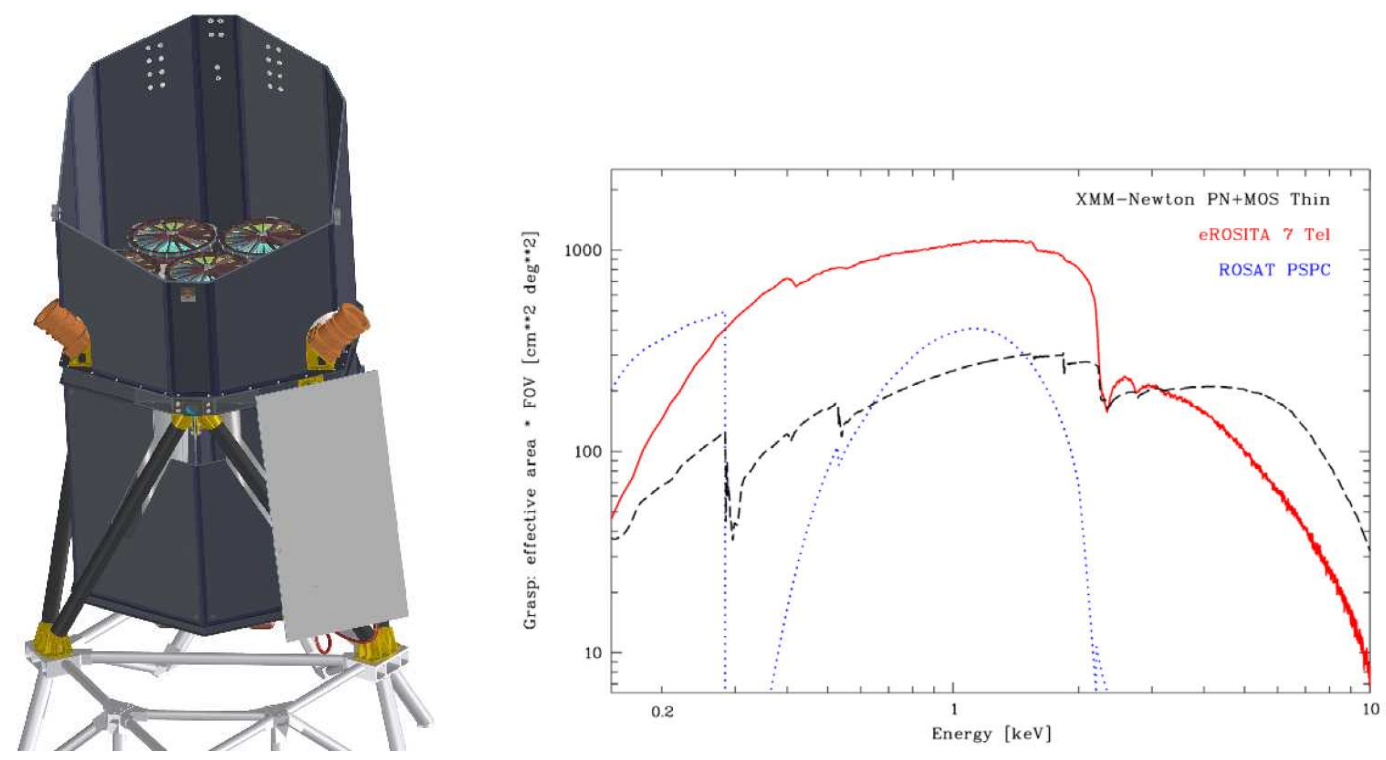

FIGURE 1. Left Panel : The eROSITA telescope on board SRG. Right Panel : The grasp of eROSITA compared with ROSAT-PSPC and XMM-Newton.

using a Soyuz-2 rocket from Bajkonur into an orbit around L2. The payload consists of the X-ray instruments eROSITA (extended ROentgen Survey with an Imaging Telescope Array) and ART-XC (Astronomical Roentgen Telescope - X-ray Concentrator).

The seven eROSITA telescopes are based on the existing design launched on the ABRIXAS mission plus an advanced version of the pnCCD camera successfully flying on XMM-Newton. In order to optimize eROSITA for the Dark Energy studies, the effective area is increased by a factor of five, the angular resolution is improved by a factor of two, and the field of view is also increased by a factor of two with respect to ABRIXAS. Such a design has been drawn to match the outcome of the most recent calls for ideas on Dark Energy observations (like e.g. by NASA, DOE, ESA, ESO and others).

Similarly to eROSITA ART-XC contains 7 telescopes working in the energy range between 6 and $30 \mathrm{keV}$. The telescopes are conical approximations of the Wolter-I geometry with CdTe detectors in their focal planes (Pavlinsky et al., 2010, this Volume)

\section{DESIGN DRIVING SCIENCE}

\section{Dark Energy}

One way to test cosmological models and to assess the origin, geometry, and dynamics of the Universe is through the study of the large-scale structures Indeed galaxy clusters are strongly correlated and thus they are good tracers of the large-scale structure on very large scales by sampling the most massive congregates of matter. The galaxy cluster population provides information on the cosmological parameters in several complementary 
ways:

1. The cluster mass function in the local Universe mainly depends on the matter density $\Omega_{m}$ and the amplitude of the primordial power spectrum $\sigma_{8}$.

2. The evolution of the mass function $f(M, z)$ is directly determined by the growth of structure in the Universe and therefore gives at the same time sensitive constraints on Dark Matter and Dark Energy.

3. The amplitude and shape of the cluster power spectrum, $\mathrm{P}(\mathrm{k})$ and its growth with time, depend sensitively on Dark Matter and Dark Energy.

4. Baryonic wiggles due to the acoustic oscillations at the time of recombination are still imprinted on the large scale distribution of clusters (i.e. in their $\mathrm{P}(\mathrm{k})$ and the Autocorrelation function) and thus can give tight constraints on the curvature of space at different epochs.

The constraints provided by the different cosmological tests with clusters are complementary in such a way, that degeneracies in the parameter constraints in any of the tests can be broken by combinations. The simultaneous constraint of $\Omega_{m}$ and $\sigma_{8}$ by combining method 1 and 3 above is one such example [9]. In addition the combination of several tests provides important consistency checks as explained below. In addition to the above applications, galaxy clusters have been used as cosmological standard candles to probe absolute distances, analogous to the cosmological tests with supernovae type Ia. The assumption that the cluster baryon fraction is constant with time combined with observations of this quantity provides constraints on Dark Matter and Dark Energy, e.g. [1]). In a very similar way, combined X-ray and Sunyaev-Zeldovich-measurements provide a mean for absolute distance measurements and constraints of the geometry of the Universe, e.g. [11].

Large, well defined and statistically complete samples of galaxy clusters (which are dynamically well evolved and for which masses are approximately known) are obvious prerequisites for such studies. A substantial progress in the field requires samples of tens to hundreds of thousands of clusters. Surveys at several wavelengths are used or planned to be used to achieve this goal. More in general, in X-ray surveys, galaxy clusters are detected by the radiation of the hot intracluster medium and X-ray observations are still the most efficient tools to select clean cluster samples. In addition X-ray observations provide accurate estimates of the cluster physical parameters. Indeed the X-ray luminosity is tightly correlated to the gravitational mass, temperature and core radius [13]. Therefore most cosmological studies involving galaxy clusters are based on X-ray surveys (e.g. [7], [8], [?], [15].

\section{INSTRUMENT}

The mirror replication technique was developed for XMM-Newton and has then been applied to the small satellite mission ABRIXAS, which had scaled the XMM-Newton telescopes down by a factor of about 4 . The ABRIXAS optical design and manufacturing process are adopted for eROSITA partially because the inner 27 mirror shells and

therefore the focal length are kept the same. The mirror system consists of 7 mirror 


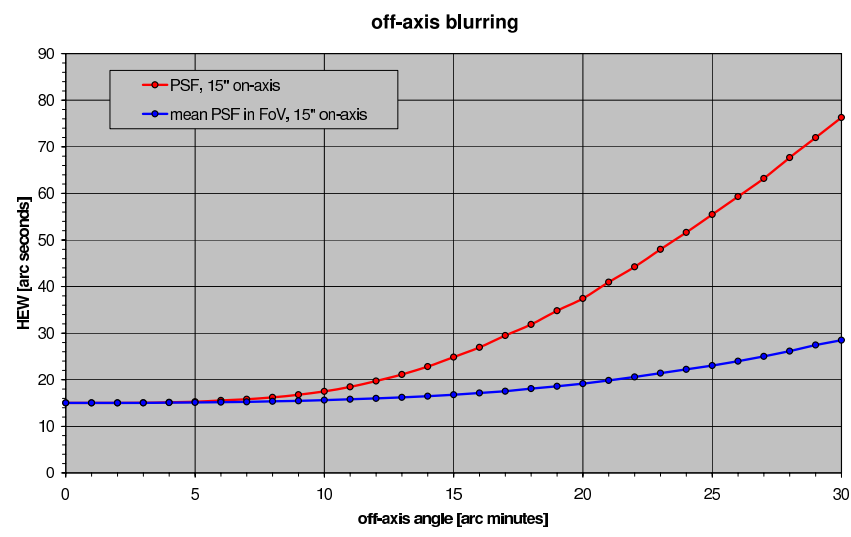

FIGURE 2. Red Curve: the PSF HEW as function of the offaxis angle in pointing mode. Blue Curve: the PSF HEW within an encircled off-axis angle during a scan. Basically the value at $30^{\prime}$, is the cumulative PSF in the survey.

modules with 54 mirror shells each and a X-ray baffle in front of each module. Unlike on ABRIXAS, the seven optical axes are co-aligned. Compared to a large single mirror system, the advantages of a multiple mirror system are: shorter focal length (reduced instrumental background) and reduced pileup when observing bright sources. This configuration allows a more compact telescope and multiple but identical cameras which automatically provides a 7-fold redundancy. The capabilities of the X-ray mirror system are described by effective area, vignetting function, and PSF. The production of the flight mirrors has already started.

The eROSITA-CCD [10] have $384 \times 384$ pixels or an image area of $28.8 \mathrm{~mm} \times 28.8$ $\mathrm{mm}$, respectively, for a field of view of $1.03^{\circ}$ diameter. The 384 channels are read out in parallel. The nominal integration time for eROSITA will be $50 \mathrm{msec}$. The integrated image can be shifted into the frame store area by less than $100 \mathrm{msec}$ before it is read out within about $5 \mathrm{msec}$. CCD together with the two CAMEX and the (passive) frontend electronics are integrated on a ceramic printed circuit board (CCD-module) and is connected to the "outer world" by a flexlead. The flight-CCDs have already been fabricated. For operation the CCDs have to be cooled down to $-80{ }^{\circ} \mathrm{C}$ by means of passive elements (heatpipes and radiator). Fluorescence X-ray radiation generated by cosmic particles is minimized by a graded shield consisting of aluminum and boron carbide. For calibration purposes, each camera housing contains a radioactive $\mathrm{Fe}^{55}$ source and an aluminum target providing two spectral lines at $5.9 \mathrm{keV}\left(\mathrm{Mn}-\mathrm{K}_{\alpha}\right)$ and $1.5 \mathrm{keV}\left(\mathrm{Al}-\mathrm{K}_{\alpha}\right)$. The mechanism ("Filter Wheel") for moving the calibration source into and out of the field of view is designed and qualified. Also the telescope structure is also qualified. The optical bench connects the mirror system and the baffles on one side with the focal plane instrumentation on the other side. Additionally it forms the mechanical interface to the $\mathrm{S} / \mathrm{C}$ bus. The flight model manufacturing is ongoing. The dimensions of the telescope structure is of the order $1.9 \mathrm{~m}$ diameter $\mathrm{x} 3.2 \mathrm{~m}$ height. The total weight of eROSITA is $735 \mathrm{~kg}$ [12]. The instrument design is shown in Figure 1. 


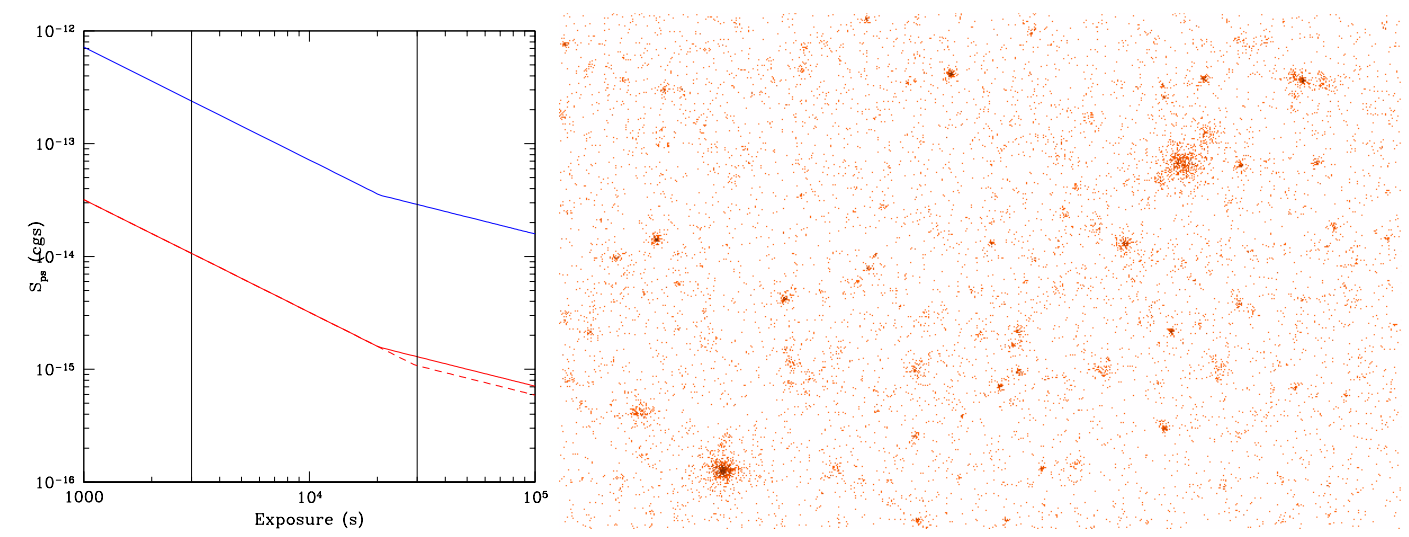

FIGURE 3. Left Panel : the $5 \sigma$ point-source sensitivity vs. exposure in the $0.5-2 \mathrm{keV}($ red $)$ and $2-10$ $\mathrm{keV}$ (blue) energy bands by assuming a PSF HEW of $30^{\prime \prime}$ and $40^{\prime \prime}$, respectively. The dashed lines is the sensitivity achieved with an average PSF-HEW of $25^{\prime \prime}$. Right Panel : A simulation of a $3 \mathrm{ks}$ observation of a $1^{\circ} \times 1.6^{\circ}$ of eROSITA in survey mode. The simulations includes cosmis+particle background, randomly distributed AGN and clusters extracted from Hydrodinamical simulations.

\section{SENSITIVITY}

Figure 1 shows the grasp of eROSITA, i.e. the product of effective area and solid angle of the field of view. The effective area of eROSITA is about twice that of one XMMNewton telescope in the energy band below $2 \mathrm{keV}$, whereas it is three times less at higher energies. This is a consequence of the small f-ratio (focal length vs. aperture) of the eROSITA mirrors. An advantage of the short focal length is also larger field of view. The eROSITA angular resolution is 15 arcsec on-axis. Due to the unavoidable off-axis degradation of a Wolter-I telescope, the angular resolution averaged over the field of view is of the order of $28^{\prime \prime}$ (Fig. 2). We will scan the entire sky for four years (ROSAT $1 / 2$ year). Therefore the eROSITA sensitivity during this all-sky survey will be approximately 30 times ROSAT. With the current scanning strategy, we expect an average exposure of $\sim 3 \mathrm{ks}$ in the all-sky survey, with two deep fields at the ecliptic poles with an exposure of the order of 20-40 ks, depending on the actual mission strategy.

We have performed simulations of the radiation environment in L2 and determined, by including the cosmic components, a background intensity of $5.63 \mathrm{cts} \mathrm{s}^{-1} \mathrm{deg}^{-2}$ and $3.15 \mathrm{cts} \mathrm{s}^{-1} \mathrm{deg}^{-2}$ in the $0.5-2 \mathrm{keV}$ and $2-10 \mathrm{keV}$ energy bands, respectively.

The $0.5-2 \mathrm{keV}$ flux limit for clusters will be, on average, of the order of $3 \times 10^{-14}$ erg cm$~_{-2} \mathrm{~s}^{-1}$ and $5 \times 10^{-15} \mathrm{erg} \mathrm{cm}^{-2} \mathrm{~s}^{-1}$ in the all-sky survey and in the ecliptic poles, respectively. In Figure 3 we plot the eROSITA $5 \sigma$ point source flux limit of the survey in the $0.5-2 \mathrm{keV}$ and $2-10 \mathrm{keV}$ energy bands as function of the exposure time. In the all-sky survey the typical flux limit will be $\sim 10^{-14} \mathrm{erg} \mathrm{cm}^{-2} \mathrm{~s}^{-1}$ and $\sim 3 \times 10^{-13} \mathrm{erg}$ $\mathrm{cm}^{-2} \mathrm{~s}^{-1}$ in the $0.5-2 \mathrm{keV}$ and $2-10 \mathrm{keV}$ energy band, respectively. At the poles we expect to reach flux limits of the order of $\sim 2 \times 10^{-15} \mathrm{erg} \mathrm{cm}^{-2} \mathrm{~s}^{-1}$ and $\sim 3 \times 10^{-14}$ erg $\mathrm{cm}^{-2} \mathrm{~s}^{-1}$ in the $0.5-2 \mathrm{keV}$ and $2-10 \mathrm{keV}$ energy band, respectively. Note that the observation will be photon limited up to exposures of $\sim 20 \mathrm{ks}$. In the $0.5-2 \mathrm{keV}$ band, the confusion limits of 1 source every 10 beams will be reached in about 20-30 ks. At this fluxes the X-ray sky is dominated by clusters and AGN, which can be separated 
with an angular resolution of $25^{\prime \prime}-30^{\prime \prime}$. The $\log N-\log S$ of clusters is well known to the proposed depth ([6], [14], [5]). The proposed survey will identify 50,000 100,000 clusters depending on the capabilities in disentangle moderately-low extended sources from AGN. Concerning the number of AGN we can use the $\log \mathrm{N}-\log \mathrm{S}$ measurement in moderately wide field surveys, like XMM-COSMOS [3], [4], to predict the detection $3-10 \times 10^{6}$ sources, up to $\mathrm{z} \sim 7-8$, depending on the detection threshold. A simulation of a 3 ks eROSITA observation of a typical extragalactic field is shown in Figure 3. Multiband optical surveys to provide the required photometric and spectroscopic redshifts are already in the planning stages, and will be contemporaneous with or precede our survey. The cluster population will essentially cover the redshift range $\mathrm{z}=0-1.3$ and will reveal all evolved galaxy clusters with masses above $3.5 \times 10^{14} h^{-1} M_{\odot}$ up to redshifts of 2. Above this mass threshold the tight correlations between X-ray observables and mass allow direct interpretation of the data.This sample size is necessary for example to precisely characterize the cluster mass function and power spectrum in at least ten redshift bins, to follow the growth of structure with time.

\section{REFERENCES}

1. Allen, S. W., Schmidt, R. W., Ebeling, H., Fabian, A. C., \& van Speybroeck, L. 2004,MNRAS, 353, 457

2. Böhringer, H., et al. 2000, ApJS, 129, 435

3. Cappelluti, N., et al. 2007, ApJS, 172, 341

4. Cappelluti, N., et al. 2009, A\&A, 497, 635

5. Finoguenov, A., et al. 2007, ApJS, 172, 182

6. Gioia I. M., Henry J. P., Mullis C. R., Voges W., Briel U. G., Böhringer H., Huchra, ApJ 533, 105 (2001)

7. Henry, P., ApJ 534, 565 (2000)

8. Henry, P., ApJ 609, 603 (2004)

9. Schuecker, P., Böhringer H., Collins C. A., Guzzo L., A\&A 398, 867 (2003)

10. Meidinger, N., Andritschke, R., Ebermayer, S., Elbs, J., Hälker, O., Hartmann, R., Herrmann, S., Kimmel, N., Predehl, P., Schächner, G., Soltau, H., Strüder, L., Tiedemann, L., "UV, X-Ray, and Gamma-Ray Space Instrumentation for Astronomy XVI". Ed. Siegmund, O.H. Proc. of the SPIE 7435, pp. 743502 (2009)

11. Molnar, S., Haiman, Z., Birkinshaw, M., Mushotzky, R.F., ApJ 601, 22 (2004)

12. Predehl, P., Andritschke, R., Bornemann, W., Bräuninger, H., Briel, U., Brunner, H., Burkert, W., Dennerl, K., Eder, J., Freyberg, M., Friedrich, P., F?rmetz, M., Hartmann, R., Hartner, G., Hasinger, G., Herrmann, S., Holl, P., Huber, H., Kendziorra, E., Kink, W., Meidinger, N., Müller, S., Pavlinsky, M., Pfeffermann, E., Roh?, C., Santangelo, A., Schmitt, J., Schwope, A., Steinmetz, M., Strüder, L., Sunyaev, R., Tiedemann, L., Vongehr, M., Wilms, J., Erhard, M., Gutruf, S., Jugler, D., Kampf, D., Graue, R., Citterio, O., Valsecci, G., Vernani, D., Zimmerman, M., "UV, X-Ray, and Gamma-Ray Space Instrumentation for Astronomy XV". Ed. Siegmund, O.H. Proc. of the SPIE 6686, 668617 (2007)

13. Reiprich, T., Böhringer, H., 2002, ApJ 567, 716

14. Rosati P, Borgani S, Norman C, ARA\&A 40, 539 (2002)

15. Vikhlinin A., Voevodkin A., Mullis C. R., VanSpeybroeck L., Quintana H., McNamara B. R., Gioia I., Hornstrup A., Henry J. P., Forman W. R., Jones C., ApJ 590, 15 (2003) 